\title{
QoS-Aware Architecture for FHMIP Micromobility
}

\author{
Nuno Vasco Lopes*, Maria João Nicolau ${ }^{\dagger}$, Alexandre Santos* \\ ${ }^{*}$ Department of Informatics, ${ }^{\dagger}$ Department of Information Systems \\ University of Minho, Braga 4710-057, Portugal \\ email: vascolopes@di.uminho.pt, joao@dsi.uminho.pt, alex@di.uminho.pt
}

\begin{abstract}
Wireless networks will certainly run applications with strict QoS requirements and so, micro-mobility protocols such as Fast Hierarchical Mobile IPv6 (FHMIP) are useful tools to accomplish this new feature. The FHMIP is an effective scheme to reduce Mobile IPv6 handover disruption, however it does not support application's QoS requirements. Therefore, in order to provide QoS guarantees for real-time applications it is necessary to develop new traffic management schemes; this implies the optimization of network mobility support and also some network congestion control. A traffic management scheme of this type should take into account the QoS requirements of handover users and should implement a Resource Management (RM) scheme in order to achieve this. In this paper, a new RM scheme for the DiffServ QoS model is proposed. This new scheme is implemented by access routers as an extension to FHMIP micromobility protocol. In order to prevent QoS degradation of the existing traffic, access routers should evaluate the impact of admitting a new Mobile Node (MN), previously to the handover. This evaluation and sequent decision on wether admitting or refusing MN's traffic is based on a Measurement-Based Admission Control (MBAC) algorithm. This architecture, that has been implemented and tested using ns-2, includes a simple signaling protocol, a traffic descriptor and exhibits an adaptive behavior to traffic QoS requirements. All the necessary measurements are aggregated by Class-ofService, thus avoiding maintaining state on the individual flows.
\end{abstract}

Index Terms-Quality of Service, mobility support, admission control, signaling.

\section{INTRODUCTION}

Providing the QoS levels suited to real-time applications needs is, in itself, a big challenge for the research community. IETF community has been working, for some years now, in order to define Internet QoS models able to meet this need but the task still challenges researchers. Integrated Services (IntServ) and Differentiated Services (DiffServ) are the primary QoS models developed within IETF. The Diffserv QoS model has also

Nuno V. Lopes was partially supported by an FCT Grant (SFRH/BD/35245/2007) been used as the QoS Model able to overcome some well known scalability and complexity problems of IntServ, pushing up complexity and processing load to border routers and keeping core routers as simple as possible. However, IntServ and DiffServ models were developed to provided QoS guarantees in wired networks, where user mobility and wireless-constrained bandwidth are not a problem.

On the other hand, current Mobile IP standard lacks on QoS provisions, on scalability, robustness and on an unified RM function. Mobile IP is a macro-mobility solution and generally is not sufficient for handling micro-mobility scenarios, where cell size is small and high frequency handovers are common. There are few proposals for micro-mobility, such as Hierarchical Mobile IP, Fast Handover, Cellular IP and HAWAII but a detailed comparation of these protocols can be found, as a survey, in P. Reinbold and O. Bonaventure paper [1]. However, micro-mobility and Mobile IP are Best-Effort (BE) and do not provide QoS guarantees, so, currently, the mobility management and the QoS models work independently. Contrary to the fixed network environments, in wireless networks mobile users can potentially change their point of attachment to the network many times during a session, thus changing to a new Access Router (nAR) that may affect the applications' QoS. Moreover, wireless links have a less predictable behavior than wired links. Therefore, when the MN changes its point of attachment, active applications on mobile should negotiate their QoS requeriments in the nAR as a part of the handover procedure. Micromobility mechanisms such as FHMIP, during handovers, use tunnels to forward packets between previous Access Router (pAR) and nARs. This helps to reduce packet losses and registration time; however this is not enough because applications should also be provisioned with appropriate QoS ensuring that packets will reach the mobile node in accordance with the QoS contract. Wireless networks are more dynamic and current cell resource availability is constantly changing, either because other users moved into the cell, or because the user leaved the cell. Therefore, user mobility will require a signalization for dynamic resource 
provisioning in order to supply adequate QoS levels to MNs on a given cell. To accomplish this objective, presented work is able to integrate: Fast Hierarchical Handovers [2] to enhance MIPv6; a signaling protocol to request services; DiffServ model for traffic differentiation; and a new Admission Control (AC) scheme to prevent QoS class deterioration. This combination of components will be optimized to work together in order to support seamless handovers for mobile users running real-time applications. In general, this architecture enhances the FHMIPv6 enabling support to QoS requirements. The operation of this architecture is based on FHMIP mobility management messages that carry QoS context values to the AC algorithm functions lying in the $\mathrm{nAR}$. The $\mathrm{AC}$ decision is done before $\mathrm{L} 2$ handover using both QoS context information and estimated Class Bandwidth needs $(\mathrm{CBw})$ on the nAR. Operationally, this QoS framework preserves the QoS working conditions for MNs already being served, because AC decision is taken before any $\mathrm{MN}$ handover. In addition, this framework does not exhibit scalability problems - because routers only need to keep reduced state information - and provides QoS support, by adjusting mobile class flows accordingly to resource availability within the nAR.

\section{RELATED WORK}

Dynamic QoS provisioning architectures can be accomplished by using signaling protocols and $\mathrm{AC}$ policies. Examples of this type of architectures for the wired network are IntServ over DiffServ, aggregate RSVP, SCORE [3], Bandwidth Brokers and more recently RM in DiffServ (RMD) [4]. In spite of the aggregation enhancement and core simplification, these QoS solutions are based on resource reservations, when enforced on mobile wireless networks, introducing an extra signaling overhead due to QoS renegotiation during handovers. Consequently, significant scalability problems may arise. Literature shows that there have been some attemps to implement and enhance the mentioned QoS frameworks in wireless networks. In [5] authors present the QoSConditionality Handover for Mobile IPv6 in order to eliminate the need for signaling protocols, using QoS Option in the hop-by-hop extension header of Binding Update (BU) messages to carry QoS context. This solution has the disadvantage that all nodes needed to be modified in order to implement this funcionality. More recently, the same authors proposed a solution [6] to provide QoS guarantees in 4G wireless networks using COPS-SLS as a signaling protocol to carry out SLS dynamic negotiation for DiffServ model with MPLS. The study [7] and [8] integrates RSVP with HMIP to provide QoS guarantees on UMTS environment. In [9] authors develop a modified RSVP to establish QoS reservations and compare its performance against the tradicional RSVP in the FMIP and HMIP mobility protocols. In [10] RMD has been used in UMTS access network and performance of Measurement-Based Admission Control (MBAC) algorithms on interior nodes have been evaluated. This solution, allowing to reduce the processing load and signaling overhead on interior nodes is similar to the IntServ over DiffServ solution.

Concluding, despite of these improvements, signaling overhead and processing load problems are not completely solved. Therefore, our approach effort has been to solve this problem with more relaxed QoS requirements i.e., soft real-time services, in order to avoid signaling overhead and Bandwidth Brokers. Moreover, as the AC is based on measurements, signaling and processing load are minimized. Finally, the establishment of QoS context on nARs before handover takes place enables to reduce the number of re-negotiations with the user.

\section{Proposed QoS Model}

\section{A. Overview}

In order to implement a QoS AC (QSAC) entity for the proposed architecture, the major design issues were: to use DiffServ mechanism as the QoS model; to select the AR (Access Router) as the critical point in the end-toend path; to define QSAC as a reduce state information entity (these entities only handle QSAC messages that contain QoS parameters). Requested QoS parameters are to be handled by a RM function, which coordinates the activities required to grant and configure resources; e.g. AC determines whether a node has sufficient resources to support the requested QoS. If QoS availability checks succeed, parameters are set in the packet classifier and scheduling in order to get the desired QoS. Interior nodes are not QSAC aware because it is assumed that they have sufficient capacity for flows that might be admitted (it is assumed that constraints reside in wireless link).

An importante aspect of this model is that it uses a scalable QoS signaling where QoS signaling states are not stored in nodes. Another important aspect of this model is the support of seamless mobility during handovers, provided by the RM function in the access network nodes. RM function is based on a method that is able to provide MBAC for flows entering a DiffServ domain and describes a system that can dynamically adjust the load within classes in access networks, in order to maintain QoS levels on the $\mathrm{nAR}$, in a proactive manner. The main advantage of MBAC is that it does not have to maintain any reservation states; a measurementbased algorithm estimates the traffic levels i.e., predicted 
resource utilization, and admits flows whose resource needs are within its availability, at the time of request. Once an admission decision is made, no record of the decision needs to be stored, it does not require prereservation state nor explicit release of reservations. The admission decision will be negative if the currently carried traffic, as characterized by an estimator, plus the requested resources for the new flow exceeds the DiffServ class capacity. Otherwise, the decision is positive. The MBAC algorithm uses prior source characterization only for incoming flows; for existing flows it uses measurements to characterize them. As in wireless networks services are typically soft real-time services and the proposed architecture intends to be scalable, only MBACs are considered. The adopted MBAC scheme does not require flow state, it only uses measures of the class-aggregated traffic for each MN. Since measurements are made on aggregates, implementation overhead is not critical [11]. Traditional AC makes decisions based on the prior traffic descriptors of the existing and new flows, while our MBAC only uses the new flow's traffic descriptor and estimates the aggregated bandwidth of the existing flows. The objective of this MBAC algorithm is to guarantee QoS requirements for new incoming flows, examined for admission, and also for previously existing flows. The misrepresentation problem of traffic descriptors may be insignificant because the algorithm relies on active on-line measurements (rather than traffic descriptors) to characterize the established traffic, what helps to limit the impact of such misrepresentation. Taking into account the heaviness of performing $\mathrm{AC}$ in all network nodes, regarding the changes and overhead introduced, AC should be left for critical points. In wireless networks, critical points are the ARs on account of wireless link constrains. MBAC is in consonance with DiffServ philosophy and with wireless networks nature because it only intends to provide soft guarantees for real-time applications. The absence of rigid QoS guarantees in wireless environment does not result into a problem, since most real-time applications in wireless networks have adaptive playback times, thus tolerate occasional packet losses and varying delays. Thereby, MBAC is sufficient to support soft real-time applications in wireless networks and may provide, in some cases, a higher link utilization [12]. So, it turns out to be one of the best options to accomplish AC on wireless networks.

\section{B. QoS Signaling}

The adopted approach in this work has been to reduce the heaviness of signaling overhead i.e., to reduce the state variables and messages in transit, preserving the scarce wireless resources and also avoiding excessive signalization caused by handovers in protocols such as RSVP and NSIS. As the architecture is based in estimations for the new flows and in measurements for the existing flows, it does not need a complex signaling protocol. For simulation purposes a simplified signaling protocol, implemented on ns-2, was used so that applications express their service requests to the network. Service requests contain a traffic descriptor describing the worst case behavior of the application traffic. This signaling protocol communicates to the edge ARs both traffic and service specification of an incoming flow. New flows use a Signaling Agent (SA) to request services to network; this SA is responsible for the delivery of all service request messages. SA-REQ (Signaling Request) messages contain the traffic description that will be the input of the RM function. The message contains two parameters: Desired Bandwidth and Class. The Signaling Agent sets the desired bandwith and classe such that each QSAC entity on path could read and interpret those parameters. If it fails to satisfy the desired QoS, the receiving Signaling Agent generates a negative SA_CONF (Signaling Confirmation) message to the SA initiator, a negative decision, and the flow is aborted. Otherwise, the receiving Signaling Agent generates a SA_CONF with a positive decision and the flow may proceed with its traffic. A two-way transaction procedure will be used to populate the QoS parameters.

\section{Mobility}

A QoS based handover management carried out at layer-2 would require a specific strategy suited to each type of wireless access network, so it is best that QoS handover is carried out at layer-3. This will allow a common framework with seamless handovers accross different access network technologies. To achieve a QoS enhancement in MIPv6 an optimized mobility management scheme with Fast (with strict delay bounds) and Smooth (with minimum packet loss) Handovers is mandatory. Combining Fast Handover and HMIPv6 enables to anticipate layer 3 handover, so that data traffic can be efficiently redirected to the MN's new location before it moves there. The hierarchical mobility management model enables the performance enhancement of Mobile IPv6 with local bindings, while using Fast Handovers helps MNs to achieve seamless mobility. Therefore, the use of both HMIPv6 and FAST Handover is crucial. The strategy adopted in order to integrate HMIPv6 and Fast Handover mechanisms has been to place MAP (Mobility Anchor Point) as the aggregation router, i.e., the first node of convergence or divergence 
depending on the direction of the data-path. Locating MAP at the aggregation router may improve the efficiency because, being it the first point of divergence, it is the best place to redirect traffic to a new path, thus saving delay and bandwidth between the aggregation router and the pAR (previous Access Router). As a conclusion, this mechanism can reduce BU signaling latency since intradomain handovers are performed locally. However, this is not enough as there is also the need to establish the QoS context that the MN had on the pAR, whenever an handover occurs. Therefore, transfering QoS contexts would facilitate other protocols to operate without the need for context restablishment. Considering that Admisssion Control mechanisms would benefit from receiving QoS context in advance, it is desired to design a new scheme that couples both the mobility management and the QoS context. When a handover at the edge of a network domain takes place, the cross-over node, MAP, plays a central role in managing the QoS context signaling establishment on the nAR.

The steps of the handover process in this new architecture are (see Figure 1):

1) When a $\mathrm{MN}$ decides to initiate a handover it sends a Router-Solicitation-for-Proxy message to the pAR, to get a new Care-of-Address. The pARs forms a HI message, containing the MAC address of the nAR and the QoS context of the MN, and sends this message to its MAP. QoS context is extracted from a Time Sliding Window Estimator (TSWE) that measures the actual CBw load on the actual AR. This per-Class QoS context is stored in a array for the HI message;

2) MAP receives the HI message containing the $M N$ MAC address and QoS context array and transfers it to the nAR;

3) nAR receives the HI message, checks for its capabilities, using its $\mathrm{AC}$ algorithm to decide what $\mathrm{MN}$ classes of flows it could accept. Additionally, if necessary, it dynamically adapts the scheduler parameters of classes with more strict QoS requirements to accommodate the incoming handover flows;

4) Next it forms a valid Care-of-Address (CoA), places it on a HAck message and returns the message to MAP;

5) MAP receives the HAck from the nAR and forwards it to the pAR;

6) The pAR receives the HAck and generates the Proxy-Router-Advertisement (PrRtAdv) message, containing the new MN CoA;

7) After $\mathrm{MN}$ receives the PrRtAdv message it sends

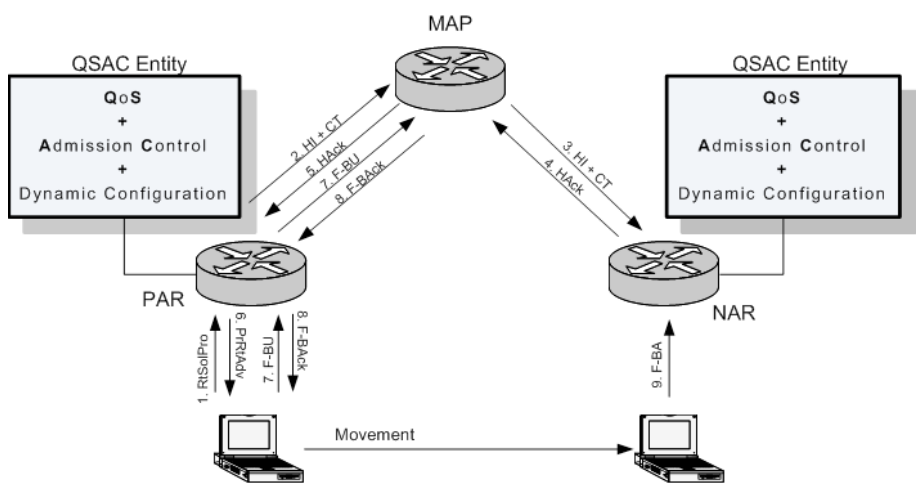

Figure 1: Handover Signaling Process

a Fast Binding Update (F-BU), via the pAR, to the MAP;

8) MAP receives F-BU message and sends a F-BAck message to $\mathrm{MN}$. The $\mathrm{MN}$ needs to wait for the FBAck messages before making the handover; this message indicates that MAP is prepared to make the tunnelling of the packets to the nAR;

9) When $\mathrm{MN}$ receives F-BAck message, first it disconnects from the pAR and then re-attaches to the nAR. At the nAR the MN sends a Fast Binding Update (F-BA), triggering the delivery of eventually delayed packets from the nAR.

\section{Resource Management Function Behavior}

$\mathrm{AC}$ algorithms limit the number of flows to be admitted into the network so that each individual flow may get the desired QoS. Therefore, they provide highquality communication by ensuring the availability of bandwith to carry load. Inelastic real-time flows, such as Voice over Internet Protocol (VoIP) or video conferencing services, can benefit from AC mechanisms. AC schemes across heterogeneous networks, based on wireless constrains, resource availability, class of service and mobile policy still remains an open issue. AC will determine whether the available resources in the network can support the requirements of $\mathrm{MN}$ when it changes to the nAR. Case there are available resources, it admits the incoming MN flow's and, if necessary, adapts the scheduler parameters to keep with the QoS requirements. Otherwise, it only rejects those flows that belong to the refused class and admits all others.

Additionaly, our algorithm will create a system that can dynamically adjust the load within classes, redistributing the $\mathrm{BE} \mathrm{CBw}$ in order to maintain the $\mathrm{QoS}$ level for priority classes, in a proactive manner. The information required, in handovers, for $\mathrm{AC}$ decision can be grouped into two categories: class information (the 
load in each class that $\mathrm{MN}$ had in $\mathrm{pAR}$, at the handover instant) and router information (the estimated class load in the nAR at the handover instant). The AR only needs to store the information of the estimated bandwidth in each DiffServ class and the aggregated traffic bandwidth for each MN (see Figure 3). The CBw estimation is the sum of the average bandwidth of the aggregated traffic with the requested bandwidth for the new flow. QoS context is extracted from a TSWE that measures the actual bandwidth load, per class, on behalf of MN. When $\mathrm{MN}$ intends to move towards a new router, the QoS context created on current AR is sent to the nAR, encapsulated on a FHMIP mobility management message (Handover Initiate). After receiving the QoS context, nAR will compare the new QoS requirements with its resource availability (see Equation 4) using the class AC that compares the requested $\mathrm{CBw}$ for the $\mathrm{MN}$ plus the estimated $\mathrm{CBw}$ in the $\mathrm{nAR}$, against the resources for that class on the nAR. If computed resources are not enough it refuses the flows on that class, otherwise admits it, with or without re-configuring scheduling parameters. Consequently, only the MN flow's that will not violate the QoS resources in the nAR will be transfered, hence ensuring acceptable levels of QoS on the AR. So, the handover of any of the MN flow's is conditioned by class resource availability in nAR. The following equations present the policy implementation on AR nodes

$$
B w=\sum_{i=1}^{D} \text { Class }_{i} \quad \text { where } B_{w} \leq C
$$

where $D$ is the number of DiffServ classes, $B w$ is the ocupied bandwidth, Class is the sum of the aggregated traffic on a given class and $C$ is the capacity of the link;

$$
\text { Class }=\sum_{j=1}^{S} \text { Session }_{i}
$$

where $S$ is the number of sessions on a given class. Equation 2 determines the used $\mathrm{CBw}$, being

$$
\text { Session }=\sum_{k=1}^{F} \text { Flow }_{i}
$$

where $F$ is the number of flows on a given session.

The QoS context to be transfered to the nAR is the measured class bandwidth utilization, named ClassCntxt. On the nAR the AC will decide, based on the Equation 4, whether to accept or reject the $\mathrm{MN}$ handover.

$$
\text { Class }_{i}+\text { ClassCntxt }_{i}<T_{i}+\Delta \text { Class }_{i}
$$

where Class is the sum of the aggregated traffic on a given class, ClassCntxt is the bandwidth required, $T$ is the maximum traffic admited in the class and $\Delta$ Class is the additional bandwidth required to fulfill QoS needs.

For new flows that want to transmit its traffic to the network, the ARs on the path have to make AC decisions based on the following policy:

$$
\text { Class }_{i}+\text { Flow }_{i}<T_{i}
$$

where Class is the sum of the aggregated traffic on a given class, Flow is the bandwidth required for the flow and $T$ is the maximum traffic to admit within that class.

\section{E. Dynamic Allocator}

This element deals with the problem of adaptive management and efficient configuration of ARs, whenever a handover occurs. In order to ensure a certain network stability, AR will use an analytical model to dynamically reconfigure the allocated bandwidth, enabling $\mathrm{CBw}$ distributions based on a bandwidth reallocation mechanism with hysteresis. The main objective is to achieve better router effectiveness by using the dynamic adaptability of the scheduler parameters; this adaption is based both on the handover QoS requirements and on the actual router state. Thereby, to dynamically adapt the ARs behavior when a handover occurs, in order to maintain the required QoS for priority classes, and, additionaly, inducing the increase of handovers acceptance, the AR should do the strict shaping of incoming flows, adjusting the allocated bandwidth to the $\mathrm{BE}$ class on behalf of priority classes. Equations 6 and 7 present the policy defined to share the uncommitted part of the BE allocated bandwidth among the priority classes.

$$
\begin{gathered}
\Delta \text { Class }_{\text {min }_{i}} \leq \Delta \text { Class }_{i} \leq \Delta \text { Class }_{\max } \\
\Delta B E_{\min } \leq \sum_{i=1}^{D} \Delta \text { Class }_{i} \leq \Delta B E_{\max }
\end{gathered}
$$

This scheme provides a class threshold, with hysteresis, that may be in different states, better than the previous deterministic handover, with no adaptability. This mechanism enables an adaptive $\mathrm{AC}$ function that reacts in accordance with the handover requirements, thus increasing the number of acceptable handovers, without compromising the global system quality (see Figure 2).

\section{Simulation Results}

\section{A. Simulation Setup}

The aforementioned architecture is almost completely implemented on the network simulator, version 2.29; the only missing element is the dynamic allocator which 


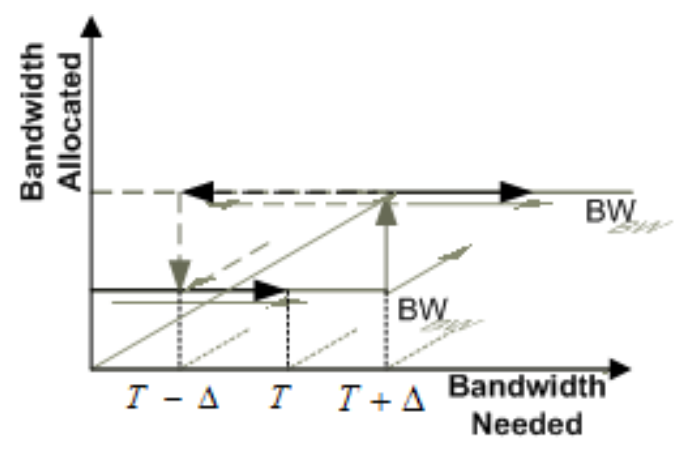

Figure 2: Allocated Class Bandwidth with Hysteresis

is still under development. Nevertheless, the results obtained with the existing implementation are very promising as presented in following sections.

Simulation has been restricted to a single DiffServ domain where the FHMIP micromobility protocol is implemented. A simple topology (see Figure 3 ) has been set up: two ARs (pAR and nAR) and two MNs (MN_1 and MN_2). Initially one $\mathrm{MN}$ is located in the pAR and the other $\mathrm{MN}$ is to be served via the nAR and both MNs are receiving traffic. Mobile nodes are receiving CBR flows, marked within different DiffServ Class, originated in fixed correspondent nodes, somewhere in a DiffServ domain. All flows start at different instants of time, within the period 0-80 seconds. MN_1 traffic includes one flow of $13 \mathrm{Kbps}$ in Class 1 , two flows of $15 \mathrm{Kbps}$ each in Class 2, five flows of 30Kbps each in Class 3 and three flows of $60 \mathrm{kbps}$ each in Class 4 , for a traffic grand total (all classes aggregated) of 373Kbps. MN_2 traffic includes one flow of $18 \mathrm{Kbps}$ in Class 1 , one flow of $30 \mathrm{Kbps}$ in Class 2, two flows of $20 \mathrm{Kbps}$ in Class 3 and two flows of $40 \mathrm{kbps}$ in Class 4, for a traffic grand total (all classes aggregated) of $168 \mathrm{Kbps}$. After 80 seconds have passed, MN_1 starts to move towards a region within the nAR scope and so all its traffic may eventually (if all accepted) move from the pAR to the nAR router.

To evaluate the proposed architecture three distinct configurations were implemented: $i$ ) without any QoS mechanisms; $i i)$ with DiffServ mechanism alone; iii) combining DiffServ and AC mechanisms. In the latter configuration when the MN intends to move to the new router, its QoS requirements (QoS context) will be submitted to a class-based AC in order to evaluate resource availability in the nAR so that only the mobile flows that belong to the accepted classes will be transfered.

\section{B. Throughput Analysis}

Despite the fact that link capacity for IEEE 802.11 was set with $1 \mathrm{Mbps}$ on $\mathrm{ns}-2$, the available bandwidth on

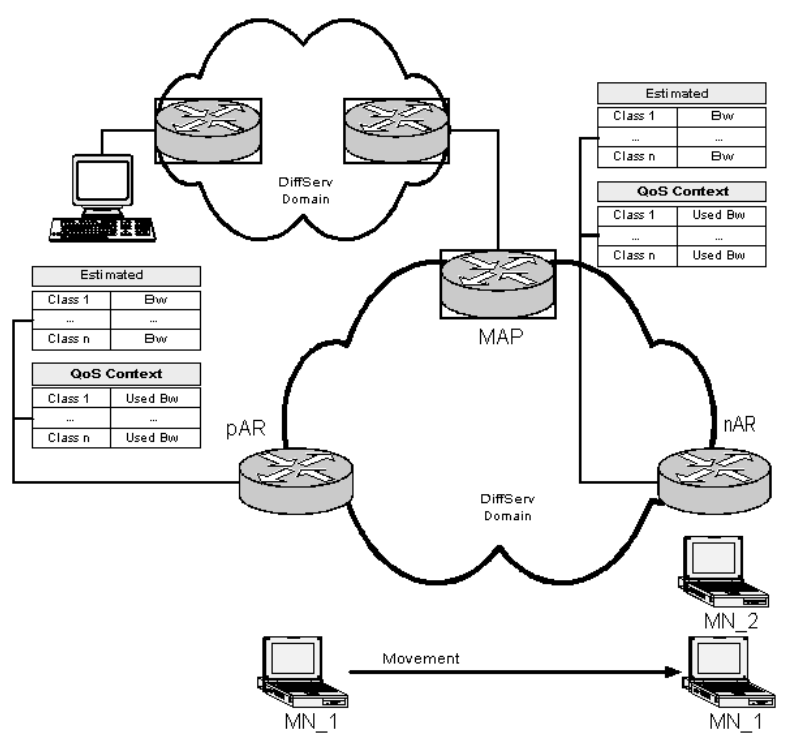

Figure 3: Architecture with per Class Tables

medium i.e., the maximum guaranteed throughput that can be transmitted between the base station and the MN without disrupting of any ongoing flow in the network, is only about $35 \%$ of the base bandwidth. This is because of its carrier sense mechanism, that whenever a node needs to send a frame, it needs to contend for medium access and it cannot transmit its frame until the medium is free.

1) S1 - Scenario without QoS mechanisms: To facilitate the analysis, traffic flows were agregated the same way as in the configurations where DiffServ classes do exist, although they have no differentiated treatment in this scenario. Figure4a shows that before handover occurs, when the radio channel reaches its maximum capacity, close to second 45 , flows reduce their throughput because they start to loose packets. After the handover, with the MN_1 in the nAR, the throughput sharply decreases for all the flows since channel must be shared by both MNs. Figure 4 illustrates that with this configuration flows are equally treated when the channel is congested.

2) S2 - Scenario with DiffServ QoS mechanism: Figure 5 shows the MNs 1 and 2 throughput when using DiffServ mechanisms. The scheduling mechanism used in DiffServ model was the Priority Queueing (PRI). Figure 5 shows a very aggressive behavior for $\mathrm{BE}$ traffic (class 4) when the channel becomes congested causing the so called traffic starvation. On the other hand the remaining and privileged classes maintain their throughput at the expense of the BE class.

3) S3 - Scenario with DiffServ and AC as QoS mechanisms: The $\mathrm{AC}$ components i.e., the measure sum algo- 


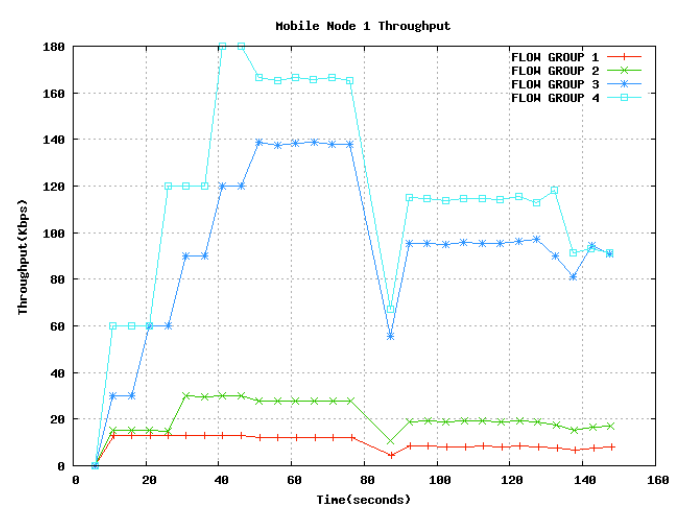

(a) S1 - Per-Class MN_1 Throughput

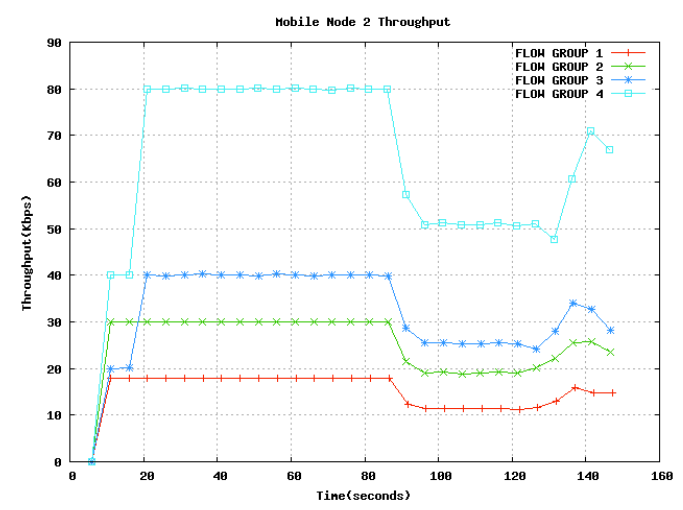

(b) S1 - Per-Class MN_2 Throughput

Figure 4: Mobile Node Throughput (no QoS Mechanisms)

rithm and TSWE have been configured with parameters of: $10 \%$ BA (Bandwidth Allocation) for Class 1, 20\% BA for Class 2 and 30\% BA for Class 3 and the remaing $40 \%$ for Class 4 (BE); 3 seconds for window size (T), 0.7 seconds for sample period (S) and 0.0625 seconds for average arrival rate estimation (W). Figure 6 shows that, even before the handover, and contrary to the precedent scenarios, when radio link limit is reached the new flows are rejected avoiding the throughput deterioration of the existing flows. At handover, AC function rejects class 3 traffic causing the flows deletion of that class. Rejection result from the fact that allocated resources for this class in nAR were not enough to acommodate the traffic of both mobiles nodes. BE traffic, class 4 , is not subject to the AC function thereby never refused. The available bandwidth for this class (BE) on the nAR is shared between MNs resulting in a throughput reduction for both nodes.

\section{Delay, Loss and Jitter Analysis}

Results show that without QoS mechanisms, after MN_1 handover takes place the delay dramatically increased to 12 seconds, for all flow groups in MN_2, with the congestion of the channel. While for scenario

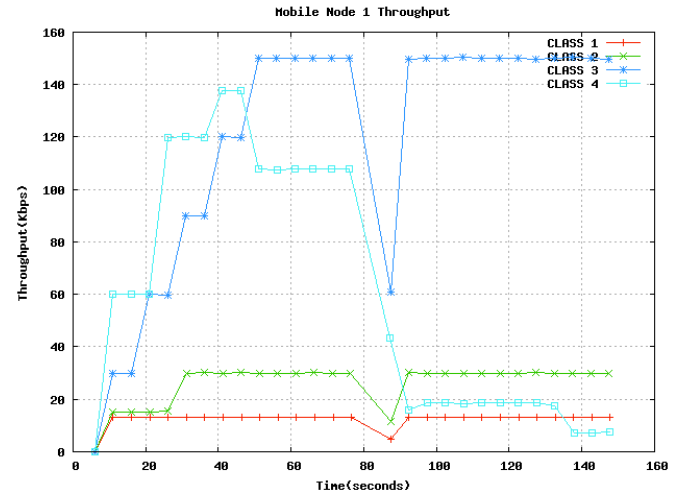

(a) S2 - Per-Class MN_1 Throughput

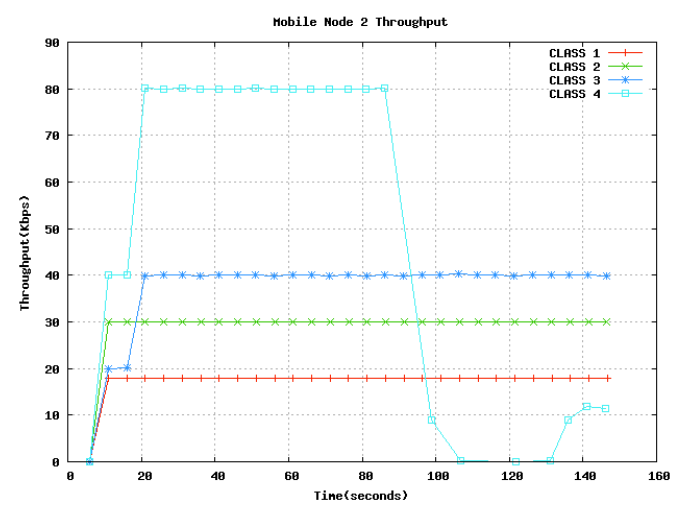

(b) S2 - Per-Class MN_2 Throughput

Figure 5: Mobile Node Throughput, using DiffServ

with DiffServ only BE traffic (Class 4) sharply climbed to 2.8 seconds whereas the remaining classes slightly grow from $25 \mathrm{~ms}$ to $30 \mathrm{~ms}$. The introduction of the AC mechanism in the simulation helps to reduce the effect that channel congestion has on delay, because it prevents the misbehavior of priority classes, limiting their throughput to the allocated bandwidth. In what concerns to losses, results show a very significant packet loss when the channel is saturated, either without QoS mechanisms or with DiffServ mechanism. DiffServ mechanism alone did not improve loss control because it does not avoid channel congestion. With DiffServ and AC, losses are substancially reduced since the amount of injected traffic is then bounded. In what regards jitter, results show a significant increase in jitter after MN_1 handover, due to the link congestion for the scenario without QoS mechanisms. In the scenario with DiffServ, jitter values for priority classes are kept limited, even when the channel is congested. BE traffic, in line with the expected in the DiffServ alone context, has its jitter severely aggravated. Scenario with DiffServ and AC reveals a significant jitter enhancement for $\mathrm{MN} \_2$ both before and after handover, when compared with the above mentioned configurations, due to $\mathrm{AC}$ supervision that 


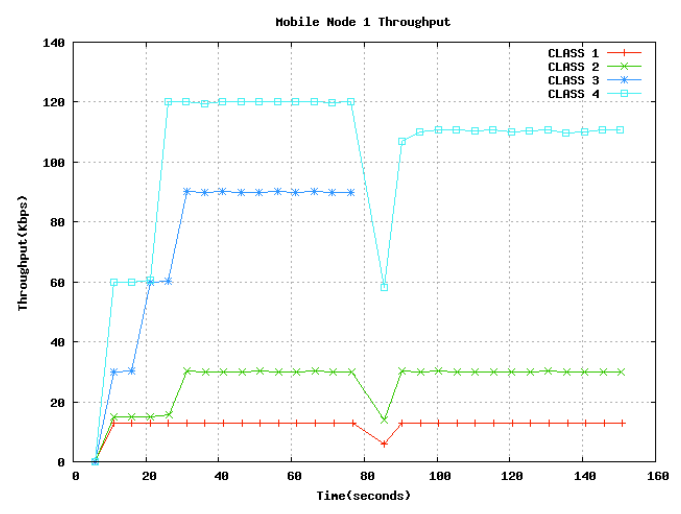

(a) S3 - Per-Class MN_1 Throughput

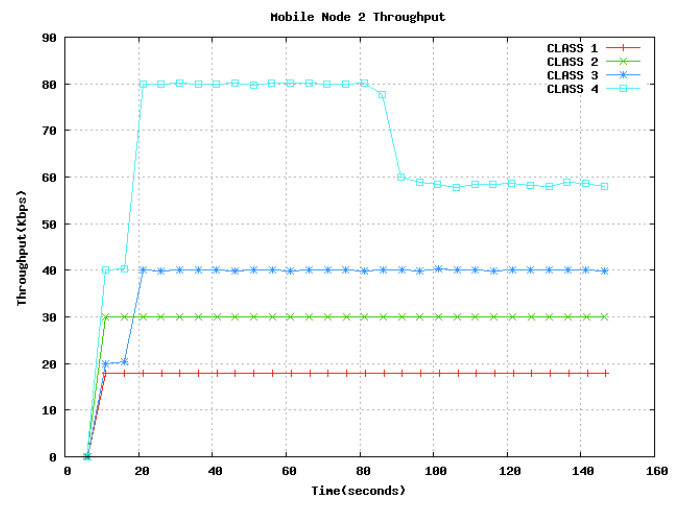

(b) S3 - Per-Class MN_2 Throughput

Figure 6: Mobile Node Throughput, using DiffServ and AC

limits the traffic on the channel. Futhermore, the priority classes almost maintain the same jitter after handover.

\section{CONCLUSiON}

This work proposes an add-on to FHMIP micromobility protocol, enabling the support for QoS. For this purpose a new Resource Management function for DiffServ model has been designed. The implemented RM function is a scalable solution based on a class MBAC algorithm. The whole architecture has been conceptualized at the network layer, in order to provide a common framework across different access tecnologies. Our scheme reduces signaling overhead because it uses an in-band message with both mobility and QoS information, avoids the congestion overload on the nAR by implementing a measure-based AC to support handover decision. Also, it provides the control of mobility with a seamless capability, allocating the mobile QoS requirements in advance on the nAR, leading to a reduction in delay, packet loss and jitter, both during handover and after it occurs. Being a measurement-based solution, it does not have to keep soft-state reservations, it offers a simple traffic descriptor and exhibits a reasonable adaptable behavior, suited to dynamic wireless environments. The
RM function implemented in the Access Routers has the ability to evaluate the impact of admitting the incoming MN flows before the MN moves to the router, thus preventing QoS deterioration of existing traffic. In future work, the handover process will be policied and will react in accordance with the handover requirements, in order to ensure a certain stability in the network. This will probably lead to a perceived QoS improvement, from the customer's point of view. Futhermore, from the network operator's point of view, this solution could also reduce network resource under-utilization. Currently, we are deploying the whole solution on the ns-2 platform.

\section{REFERENCES}

[1] P. Reinbold and O. Bonaventure, "A survey of ip micro-mobility protocols," Technical Report 2002-06, Infonet, March 2002.

[2] R. Koodli, "Mobile IPv6 Fast Handovers." RFC 5568 (Proposed Standard), July 2009.

[3] I. Stoica and H. Zhang, "Providing guaranteed services without per flow management," SIGCOMM Comput. Commun. Rev., vol. 29, no. 4, pp. 81-94, 1999.

[4] A. Bader, L. Westberg, G. Karagiannis, C. Kappler, and T. Phelan, "Rmd-qosm - the resource management in diffserv qos model," IETF Internet draft draft-ietf-nsis-rmd-12, USA, November 2007.

[5] C. Liu, D. Qian, Y. Liu, and K. Xiao, "A framework for endto-end differentiated services qos context transfer in mobile ipv6," in Proc. First IEEE and IFIP International Conference in Central Asia on Internet (D. Qian, ed.), pp. 466-475, 2005.

[6] C. Liu, Y. Liu, D. Qian, and M. Li, "An approach of end-toend diffserv/mpls qos context transfer in hmipv6 net," in ISADS '07: Proceedings of the Eighth International Symposium on Autonomous Decentralized Systems, (Washington, DC, USA), pp. 245-254, IEEE Computer Society, 2007.

[7] L. Sanchez, L. Sanchez, and P. Ruiz, "Qos and micromobility coupling integrated with umts," in Proc. 15th IEEE International Symposium on Personal, Indoor and Mobile Radio Communications PIMRC 2004 (P. Ruiz, ed.), vol. 2, pp. 763768 Vol.2, 2004.

[8] L.-A. Galindo-Sanchez and P.-M. Ruiz-Martinez, "Qos and micromobility coupling: Improving performance in integrated scenarios," UPGRADE, vol. VI, April 2005.

[9] A. Belhoul, Y. A. Şekercioğlu, and N. Mani, "Mobility protocols and rsvp performance in wireless ipv6 networks: shortcomings and solutions," Wireless Communications and Mobile Computing, vol. 8, no. 9, pp. 1183-1199, 2008.

[10] H. E. Allali, G. J. Heijenk, A. Lo, and I. G. M. M. Niemegeers, "A measurement-based admission control algorithm for resource management in diffserv ip networks," Proceedings 17th Annual IEEE International Symposium on Personal, Indoor and Mobile Radio Communications (PIMRC'06), pp. 1-5, September 2006.

[11] S. Jamin, S. J. Shenker, and P. B. Danzig, "Comparison of measurement-based admission control algorithms for controlled-load service," in Proc. IEEE Sixteenth Annual Joint Conference of the IEEE Computer and Communications Societies INFOCOM '97, vol. 3, pp. 973-980 vol.3, 1997.

[12] J. K. Abbas Jamalipourn, "Measurement-based admission control scheme with priority and service classes for application in wireless ip networks," INTERNATIONAL JOURNAL OF COMMUNICATION SYSTEMS, vol. 16, pp. 535-551, May 2003. 